\title{
DEPENDÊNCIA DA TRAJETÓRIA, IRREVERSIBILIDADE E O PAPEL DA HISTÓRIA NA SELEÇÃO DE TECNOLOGIAS
}

\author{
Antonio Luis Licha ${ }^{1}$
}

\section{INTRODUÇÃO}

$\mathrm{Na}$ última década alguns economistas têm procurado incorporar em suas teorias a idéia de que "a história importa". Uma forma de fazer a incorporação é pelo conceito de dependência da trajetória (path-dependence). $\mathrm{O}$ conceito diz respeito ao fato que certos processos mostram bifurcações provocadas por pequenos eventos circunstanciais (históricos) que imprimem primeiro uma mudança de direção que se torna gradualmente irreversível. ${ }^{2}$ Essa irreversibilidade na trajetória adotada é chave para o conceito de dependência da trajetória já que uma vez que o processo econômico entrou numa certa rota existem forças internas que fazem que não seja possível abandoná-la espontaneamente. Dessa forma a estrutura econômica torna-se crescentemente rígida, sendo mais difícil de ser alterada.

Esses conceitos são aplicados a vários contextos, como à seleção de tecnologias, à evolução de instituições, ao crescimento econômico e a mercados financeiros. ${ }^{3}$ Neste capítulo, nos limitaremos a utilizar esses conceitos para analisar como algumas tecnologias são escolhidas pelos agentes econômicos e selecionadas pelo mercado.

1 IE - UFRJ <E-mail: licha@ie.ufrj.br>

2 Em inglês, isso denomina-se lock-in, o que quer dizer que o sistema está bloqueado numa certa trajetória.

3 Uma resenha desta literatura pode ser encontrada em David (2000). 
Que é o que faz que a escolha de uma tecnologia mostre dependência da trajetória e irreversibilidade? Uma condição suficiente é de que existam fortes complementaridades no comportamento dos agentes, de forma que seja vantajoso adotar uma tecnologia porque ela já foi adotada antes por outros agentes. Em outros termos, devem existir retornos crescentes (mecanismos de realimentação que reforcem as escolhas tecnológicas realizadas num certo momento) associados a essas tecnologias: os benéficos da adoção das mesmas aumentam à medida que aumenta o número de adotantes. Assim, spillovers tecnológicos podem fazer que as decisões dos agentes sejam o suficientemente interdependentes para que os processos apresentem uma multiplicidade de resultados.

Segundo vários autores (por exemplo, ARTHUR, 1994, p. 28), a existência de retornos crescentes exige mudanças nos métodos de análise utilizados. A análise estática torna-se insuficiente, já que existem múltiplas soluções ou resultados possíveis para a evolução de um certo processo. A razão disso já deve ser bastante óbvia: se pequenos choques mudam a trajetória de forma irreversível, e esses choques são circunstanciais ou aleatórios, não há como predizer que caminho será efetivamente seguido pelo processo estudado. No tema que nos ocupa, a seleção de tecnologia, a dependência da trajetória implica que não é possível predizer ex ante que tecnologia predominará ou será finalmente selecionada pelos agentes. Potencialmente há equilíbrios (ou trajetórias de desequilíbrio) múltiplos, e não temos como saber qual dessas possibilidades tornar-se-á efetiva. ${ }^{4}$ Para entender como uma configuração é selecionada é necessário seguir o processo "passo a passo", analisando como certos eventos históricos alteram uma trajetória. Por esta razão, tratando-se de retornos crescentes, é preciso explorar a dinâmica de seleção, e colocar o movimento do sistema no centro da análise. Os resultados não podem ser definidos ex ante, sem conhecer o caminho específico seguido pelo processo ao longo de diversos períodos.

O método proposto por Arthur (1994) para estudar a dinâmica do sistema é analisar, por um lado, as suas tendências de longo prazo. Em outras palavras, ele analisa a configuração que emerge após o transcurso de um número suficientemente elevado de períodos, de forma a eliminar o efeito das flutuações de curto prazo (a chamada dinâmica transitória). Essas últimas, pela sua vez, podem ser analisadas usando modelos de simulação. Neste

$4 \quad$ No mesmo sentido, ver Durlauf (1993). 
capítulo, trataremos apenas dos resultados de longo prazo do processo, deixando de lado a análise da dinâmica transitória.

O capítulo é organizado da seguinte forma. Na próxima seção são apresentadas as definições dos principais conceitos relativos à dependência da trajetória. Na seção 2 estuda-se em que condições é possível esperar que um sistema mostre retornos crescentes. Na seção 3 o conceito é ilustrado usando exemplos históricos. Na seção 4 oferece-se uma análise dessas idéias a partir de um modelo proposto por B. Arthur (1989). Na seção 5 discutimos as principais implicações de política tecnológica que se derivam da análise proposta. $\mathrm{Na}$ última seção são apresentadas algumas reflexões finais. No anexo apresentamos detalhes técnicos das principais propriedades do processo analisado.

\section{DEFINIÇÕES}

A seguir, oferecem-se algumas definições simples dos principais conceitos usados neste capítulo. ${ }^{5}$

- Dependência da trajetória: se a evolução de um processo está condicionada pela sua própria história então ele é dependente da trajetória. ${ }^{6}$ Não é possível entender o resultado do processo sem considerar os eventos específicos que ocorreram em distintos momentos no passado. Neste caso, um mesmo processo pode dar lugar a múltiplas estruturas no longo prazo em função dos eventos históricos acontecidos ${ }^{7}$

- Irreversibilidade: se quando uma certa estrutura é alcançada o processo não pode sair dela de forma endógena (sem intervenção externa) então existe uma certa rigidez estrutural e o processo está bloqueado (lock-in) ${ }^{8}$

- Retornos crescentes: emergem de um processo virtuoso no qual a expansão do uso de um certo fator faz com que se torne cada vez mais

5 Definições mais técnicas dos conceitos usados podem ser encontradas nas notas de rodapé.

De forma precisa, dependência da trajetória faz referência às propriedades de processos dinâmicos não-ergódicos nos quais existem mecanismos (fortes) de realimentação positiva.

7 Algumas vezes é colocado que esses processos apresentam equilíbrios múltiplos. O conceito de equilíbrio é entendido como um atrator de longo prazo, ou seja, uma configuração que só é alcançada de forma assintôtica.

8 Essa estrutura representa um estado absorvente para o processo já que constitui uma configuração irreversível. 
vantajoso usá-lo. Esse fator pode ser um insumo, um produto, uma instituição ou uma tecnologia. Os retornos crescentes observam-se principalmente em atividades intensivas em conhecimentos. Por exemplo: quanto maior a difusão de um certo software computacional, maiores as possibilidades de interação entre usuários, de investimento e de melhoramento do software, o que na sequiência leva a um aumento do número de usuários e assim sucessivamente. Pelo contrário, o uso de certos insumos mostra retornos decrescentes, especialmente se consideramos recursos naturais. Imaginemos o caso do carvão mineral. À medida que aumenta o uso do carvão como fonte de energia na indústria, minas cada vez mais afastadas, e mais difíceis de explorar, terão de ser incorporadas ao processo de produção. Isso encarece o recurso e limita o uso de carvão como fonte de energia, enquanto que outras fontes se tornam mais vantajosas (energia hidráulica ou petróleo, por exemplo). No longo prazo, distintas fontes energéticas deverão coexistir e participar do mercado energético, já que os retornos decrescentes não permitem que nenhuma das fontes se torne exclusiva, expulsando as outras. ${ }^{9}$

\section{FONTES DE RETORNOS CRESCENTES}

Vejamos o caso dos retornos crescentes na escolha de uma certa tecnologia. À medida que mais agentes usam essa tecnologia (a sua participação na estrutura tende a aumentar), existe uma tendência para que novos agentes a adotem. A vantagem de usar a tecnologia aumenta pari passu com o número de usuários, o que indica a existência de algum mecanismo de realimentação positiva. Segundo Arthur (1994), podemos considerar cinco fontes básicas de retornos crescentes na escolha de tecnologias: ${ }^{10}$

- Economias de escala: Surgem de custos fixos ou de organização na produção das tecnologias. Trata-se de uma vantagem bastante óbvia. O custo fixo unitário resulta de dividir o custo fixo total pela quantidade produzida e, como por definição o custo fixo é constante, o custo fixo unitário cai com o aumento da produção. Por exemplo, quando a tecnologia é envolvida num produto, como na Tecnologia Polaroid, ela é mais atrativa em preço quando mais adotada;

9 Como foi visto anteriormente, isso também tem muita importância para a teoria do crescimento e da concentração regional da produção.

10 Para North (1990, cap. XI) estes mecanismos auto-reforçadores podem ser aplicados à escolha de instituições (entendidas como regras, normas, etc.). 
- Aprendizado: Uma certa tecnologia raramente permanece estática. Pelo contrário, tende a ser adaptada e melhorada de forma gradual e contínua. Quanto mais uma tecnologia é produzida e usada, maior a experiência acumulada de produtores e usuários, maiores as informações que circulam sobre a tecnologia, maior o domínio que sobre ela adquirem as pessoas (individualmente ou em equipes), e maiores serão, portanto, as possibilidades de introduzir melhoras. Quando o aprendizado ocorre principalmente durante a produção, em função da experiência e dos esforços acumulados, chama-se de "aprender fazendo". Quando ele deriva principalmente de informações sobre o desempenho da tecnologia, proporcionada pelos usuários, denominase "aprender usando". Por exemplo, a indústria aeronáutica registra esses tipos de aprendizado de forma particularmente intensa. Um novo desenho de avião, como o DC-8, ganha consideravelmente em capacidade de carga e de passageiros, em eficiência nos motores e em aerodinâmica, quando as linhas aéreas começam a utilizá-lo e a sua produção aumenta;

- Coordenação: Existe coordenação quando a decisão de um agente é complementar com as decisões dos outros. Isto pode acontecer quando:

1. uma tecnologia mais utilizada gera mais contratos e atividades complementares que permitem sua extensão a uma variedade de aplicações específicas. Um exemplo, é a tecnologia do petróleo que vai da extração, às refinarias e à distribuição dos seus derivados;

2. cooperar com as tecnologias de outros agentes tem a vantagem de que o predomínio de uma certa tecnologia gera expectativas de que ela permanecerá, reduzindo a incerteza de sua continuidade no futuro. Assim, para agentes com aversão ao risco a adoção de uma tecnologia é mais atrativa se é mais usada.

- Externalidades de rede: Algumas tecnologias oferecem vantagens se seus adotantes funcionam de forma conjunta, como nas redes de usuários. Já foi mencionado o caso dos softwares de computação. Um outro exemplo é a tecnologia de videocassetes. Quanto maior o número de usuários de um certo sistema, como o VHS, maior a produção de fitas para equipamentos que usam esse sistema, e maiores as vantagens de tornar-se usuário do sistema. O sucesso presente de uma tecnologia se explica pelo seu sucesso no passado. O mesmo vale para certas instituições. As pessoas dirigem pela direita no Brasil, e pela esquerda na Inglaterra, pela única razão de que a maior parte das pessoas já adotou essa convenção em cada país. Outro exemplo é dado pela construção de relógios. Eles são construídos de tal forma que os ponteiros giram no "sentido horário", pela simples razão de que existe uma convenção dominante sobre qual seria esse sentido. Mas no passado, muitos relógios foram construídos com as agulhas movimentando-se em sentido oposto; 
- Inter-relações tecnológicas: Freqüentemente, quando uma tecnologia é mais adotada, também se expandem outras sub-tecnologias e produtos que são parte da infra-estrutura que a tecnologia requer para funcionar eficientemente. Por exemplo, a tecnologia da gasolina tem uma vasta infraestrutura de refinarias, postos de gasolina e auto-partes que se baseiam nela. Isto promove uma vantagem em relação a outras tecnologias menos adotadas, por exemplo, o álcool, devido a que elas não dispõem da infra-estrutura necessária para seu desenvolvimento.

A seguir, mostraremos alguns exemplos de tecnologias que exibem retornos crescentes.

\section{ILUSTRAÇÕES}

Existem casos clássicos na literatura sobre seleção de tecnologias que apresentam as propriedades destacadas acima. A seguir, apresentamos casos de padronização de tecnologias que não necessariamente foram as mais eficientes do ponto de vista dinâmico. ${ }^{11}$

- O teclado da máquina de escrever (DAVID, 1985). O atual teclado (chamado de QWERTY) foi elaborado por volta de 1870 e popularizado pelas máquinas de escrever de E. Remington alguns anos depois. As pessoas foram treinadas e se adaptaram a essa estrutura durante anos. $\mathrm{O}$ aprendizado dos usuários levou a que esse teclado dominasse qualquer inovação de desenho, ainda que ela tivesse um desempenho superior. Por exemplo, nos anos trinta, A. Dvorak desenhou um teclado (chamado de DSK) que ao ser testado nos anos quarenta mostrou ter um desempenho superior (permitia uma velocidade maior e induzia menos erros na digitação). Mas os hábitos desenvolvidos levaram a que a estrutura da tecnologia de teclados ficasse bloqueada nos teclados QWERTY: essa estrutura mostrou um alto grau de irreversibilidade;

- Os motores dos carros (ARTHUR, 1994). A tecnologia disponível para a fabricação de automóveis a partir de 1890 oferecia várias alternativas para o desenvolvimento dos motores, especialmente com relação ao combustível. O uso da gasolina era uma das opções em 1895, mas existiam alguns problemas: era perigosa e o motor requeria partes mais numerosas e

11 Cowan e Gunby (1996), David e Bunn (1988) e Foray e Grübler (1990) apresentam outros estudos empíricos que mostram a existência de dependência da trajetória na concorrência de tecnologias. 
sofisticadas que o que utilizava vapor. No período entre 1890-1920 foram desenvolvidos, dependendo da experiência do fabricante em engenharia, carros com motores a vapor, gasolina e elétricos. Mas uma série de circunstâncias, como uma competição de carros em 1895 que influenciou alguns construtores como Ransom Olds a mudar do vapor para a gasolina e uma doença nos cavalos em 1914 provocada pela água lançada pelos motores a vapor, deram à gasolina uma liderança decisiva. Não é possível saber hoje se os carros com motor a vapor ou motor elétrico seriam superiores ou não aos carros que usam gasolina. Mas vale a pena lembrar que esse é um debate recorrente na literatura da engenharia mecânica;

- Os reatores nucleares (COWAN, 1990). No começo dos anos cinqüenta existiam várias alternativas para gerar energia elétrica a partir da construção de centrais nucleares. Entre elas, reatores que usam água-leve, refrigerados a gás, que usam água-pesada e refrigerados com sódio. Os reatores com água-leve tomaram conta nos oitenta de $100 \%$ das instalações americanas e de quase $80 \%$ do mercado mundial. Eles foram originalmente adaptados de uma unidade compacta desenhada para propulsar o primeiro submarino nuclear americano (o USS Nautilus) em 1954. Uma série de circunstâncias, entre as quais o papel da marinha americana nos primeiros contratos de construção do submarino nuclear (realizados pelo Almirante Rickover dentro do Programa Euratom) e as articulações políticas dentro da National Security Council, atuaram a favor da água leve. $\mathrm{O}$ aprendizado e a experiência obtida na construção das usinas nucleares, levaram a que a meados dos anos sessenta o mercado ficasse bloqueado nessa tecnologia. Mas a literatura na área de engenharia argumenta que, ceteris paribus, o reator refrigerado a gás (gascooled design) teria tido um desempenho superior;

- Os videocassetes (ARTHUR, 1994). Como vimos, a tecnologia de videocassetes exibe externalidades de rede, no sentido que o predomínio no mercado de uma certa tecnologia incentiva a produção de filmes para essa tecnologia. Nos oitenta, o aparecimento de dois concorrentes, Sony Betamax e VHS, levou a uma disputa pela liderança na participação no mercado, já que isto significava a sobrevivência de uma tecnologia, e a conseqüente exclusão da outra. Apesar de Betamax ser tecnicamente superior a VHS, segundo afirmações de especialistas da indústria, um conjunto de circunstâncias externas levou a que a tecnologia VHS tomasse a dianteira e ocupasse 100\% do mercado, gerando um tipo de ineficiência de longo prazo. 


\section{UM MODELO}

Apresentamos agora o problema da seleção de tecnologias no contexto de um modelo econômico simplificado. ${ }^{12} \mathrm{O}$ modelo tem um número grande de agentes e duas tecnologias. Mostraremos que a proporção de uso de cada uma das tecnologias flutua no começo do processo de seleção mas depois surge uma tendência clara à concentração, onde uma tecnologia é selecionada e a outra excluída.

Consideremos que existem duas tecnologias (A e B) e um número grande (infinito) de agentes econômicos podem escolher entre elas. Existem dois tipos de agentes, os agentes tipo $\mathrm{R}$ e os agentes tipo $\mathrm{S}$, com preferências diferentes, sendo inicialmente igual o número de agentes de cada tipo. Os agentes de tipo R têm preferências naturais por A, e os de tipo S por B. ${ }^{13}$

$\mathrm{O}$ retorno esperado de escolher uma certa tecnologia é V. Dada a hipótese de retornos crescentes, $\mathrm{V}$ aumenta a medida que mais agentes adotam essa tecnologia. Assim, por exemplo, as pessoas podem escolher equipamentos VHS ou Beta Max, mas o retorno esperado da escolha dependerá do número de pessoas que já adotou cada um desses sistemas.

Seja $\mathrm{n}_{\mathrm{A}}$ e $\mathrm{n}_{\mathrm{B}}$ o número de adotantes de $\mathrm{A}$ e $\mathrm{B}$, respectivamente, e $\mathrm{n}$ $\left(n=n_{A}+n_{B}\right)$ o número total de escolhas realizadas. Durante o processo de escolha acontecem "pequenos eventos históricos" (dados por determinações políticas, culturais e históricas externas à análise do modelo), que afetam a escolha dos agentes, mas que são desconhecidos para os agentes de tipo R e S. Assim, o poder de discernimento dos agentes em relação ao futuro é limitado, já que eles não têm como conhecer a ordem futura de escolha de A e B. ${ }^{14}$ Consideremos que:

12 O modelo é uma versão simplificada do modelo apresentado por B. Arthur (1989). Outro modelo (mais complexo) é apresentado por Milgrom, Qian e Roberts (1991). O modelo proposto pode ser chamado de evolucionário pois enfatiza as propriedades emergentes da estrutura de longo prazo e o caráter auto-organizativo do processo. Os modelos evolucionários consideram uma dinâmica de "ordem através de flutuações" como um paradigma para a interpretação de sistemas complexos não-lineares que enfatizam as propriedades de mecanismos auto-reforçadores e de auto-organização fora do equilíbrio. Esses sistemas são sensíveis a perturbações iniciais (pequenas) e apresentam múltiplos padrões comportamentais a longo prazo. A acumulação de pequenas perturbações iniciais (no redor de estados instáveis) leva o sistema para um destes padrões, selecionando a estrutura para o qual o sistema eventualmente tende. Neste sentido ver Dosi e Kaniovski (1994).

13 A preferência natural do agente mostra sua preferência sem considerar as decisões dos outros agentes.

14 No ambiente seletivo proposto existe racionalidade limitada por parte dos agentes. 
- $\quad$ os agentes de tipo $\mathrm{R}$ e $\mathrm{S}$ conhecem o número de pessoas que já adotou cada tecnologia;

- os agentes são "míopes", pois projetam o futuro a partir do acontecido no passado.

Os retornos esperados associados à adoção de tecnologia por parte de cada agente, são dados pela seguinte matriz de lucros:

Tabela 1 - MATRIZ DE LUCROS

\begin{tabular}{|c|c|c|}
\hline Agentes & $\mathrm{A}$ & $\mathrm{B}$ \\
\hline $\mathrm{R}$ & ${ }_{\mathrm{R}} \mathrm{V}_{\mathrm{A}}=\mathrm{a}_{\mathrm{R}}+\mathrm{rn}_{\mathrm{A}}$ & ${ }_{\mathrm{R}} \mathrm{V}_{\mathrm{B}}=\mathrm{b}_{\mathrm{R}}+\mathrm{r} \mathrm{n}_{\mathrm{B}}$ \\
$\mathrm{S}$ & ${ }_{\mathrm{S}} \mathrm{V}_{\mathrm{A}}=\mathrm{a}_{\mathrm{S}}+\mathrm{s} \mathrm{n}_{\mathrm{A}}$ & ${ }_{\mathrm{S}} \mathrm{V}_{\mathrm{B}}=\mathrm{b}_{\mathrm{S}}+\mathrm{s} \mathrm{n}_{\mathrm{B}}$ \\
\hline
\end{tabular}

Na tabela r, s, a $\mathrm{R}_{\mathrm{R}}, \mathrm{b}_{\mathrm{R}}, \mathrm{a}_{\mathrm{S}}$ e $\mathrm{b}_{\mathrm{S}}$ são parâmetros e:

- $\quad{ }_{\mathrm{R}} \mathrm{V}_{\mathrm{A}}$ : retorno esperado pela adoção da tecnologia A por parte de um agente de tipo $\mathrm{R}$,

- ${ }_{\mathrm{R}} \mathrm{V}_{\mathrm{B}}$ : retorno esperado pela adoção da tecnologia $\mathrm{B}$ por parte de um agente de tipo $\mathrm{R}$,

- ${ }_{\mathrm{S}} \mathrm{V}_{\mathrm{A}}$ : retorno esperado pela adoção da tecnologia A por parte de um agente de tipo $\mathrm{S}$,

- ${ }_{\mathrm{S}} \mathrm{V}_{\mathrm{B}}$ : retorno esperado pela adoção da tecnologia $\mathrm{B}$ por parte de um agente de tipo $\mathrm{S}$,

- $r>0$ e $s>0$, pois existem retornos crescentes,

- $\mathrm{a}_{\mathrm{R}}>\mathrm{b}_{\mathrm{R}}$ e $\mathrm{b}_{\mathrm{S}}>\mathrm{a}_{\mathrm{S}}$, o que resulta das chamadas preferências naturais: agentes $\mathrm{R}(\mathrm{S})$ tendem a preferir a tecnologia $\mathrm{A}(\mathrm{B})$ (mais sobre isso embaixo).

A matriz de lucros proporciona os ganhos esperados por cada agente quando decide que tecnologia usar. Assim, se o agente $\mathrm{R}$ elege a tecnologia A, ele espera ganhar ${ }_{R} V_{A}$, representado no quadrante superior esquerdo. ${ }_{R} V_{A}$ consiste num certo valor fixo, $\mathrm{a}_{\mathrm{R}}$, e um certo valor que aumenta com o número de usuários da tecnologia $\mathrm{A}, \mathrm{r}_{\mathrm{A}}$. E fácil ver que como $\mathrm{r}$ é positivo, o retorno de escolher a tecnologia $\mathrm{A}$ aumenta com o número de usuários de $\mathrm{A}, \mathrm{n}_{\mathrm{A}}$, e esse aumento é tanto maior quanto maior o valor do parâmetro r. Se, pelo contrário, ele escolhe a tecnologia B, o ganho estará também dado por um valor fixo $\left(b_{R}\right)$ mais um valor que depende do número de adotantes, neste 
caso, da tecnologia $\mathrm{B}\left(\mathrm{r}_{\mathrm{B}}\right)$. O mesmo raciocínio vale com relação aos agentes de tipo $\mathrm{S}$. Obviamente, os agentes escolhem a tecnologia que oferece os maiores retornos esperados em cada momento.

Assim, os agentes de tipo R começam escolhendo a tecnologia A, dada sua preferência natural. Isso fica claro se imaginamos que no momento zero $\mathrm{n}_{\mathrm{A}}=\mathrm{n}_{\mathrm{B}}$. $\mathrm{O}$ número de adotantes não faz diferença, e portanto, o que importa na hora da escolha é o valor fixo, $a_{R}$ ou $b_{R}$. Deve-se lembrar que definimos $a_{R}>b_{R}$, o que apenas reflete o suposto que a preferência natural de $\mathrm{R}$ é a tecnologia A . Mas os agentes $\mathrm{R}$ mudam sua escolha para $\mathrm{B}$ se o retorno esperado de $\mathrm{B}$ supera o de $\mathrm{A}$. Isso pode acontecer quando existe um número de adotantes de $\mathrm{B}$ muito maior do que de $\mathrm{A}$. Formalmente, eles mudam se ${ }_{R} V_{B}>{ }_{R} V_{A}$ o que acontece se $n_{B}$ é o suficientemente maior que $n_{A}$. Pode-se ver que os agentes de tipo $\mathrm{R}$ deslocam sua escolha de A para $\mathrm{B}$ se:

$$
\mathrm{n}_{\mathrm{A}}-\mathrm{n}_{\mathrm{B}}<\Delta \mathrm{R}
$$

onde $\Delta \mathrm{R} \equiv\left(\mathrm{b}_{\mathrm{R}}-\mathrm{a}_{\mathrm{R}}\right) / \mathrm{r}$. Em outros termos, se $\mathrm{n}_{\mathrm{B}}$ supera $\mathrm{n}_{\mathrm{A}}$, de forma que o aumento dos retornos esperados de $\mathrm{B}$ compensam a preferência natural dos agentes de tipo R, eles mudam sua escolha.

De forma similar, os agentes de tipo $\mathrm{S}$ mudam suas escolhas de $\mathrm{B}$ para A se os retornos de A superam os de B. Isto acontece $\mathrm{se}_{\mathrm{S}} \mathrm{V}_{\mathrm{A}}>{ }_{\mathrm{S}} \mathrm{V}_{\mathrm{B}}$, ou seja, se:

$$
\mathrm{n}_{\mathrm{A}}-\mathrm{n}_{\mathrm{B}}>\Delta \mathrm{S}
$$

onde $\Delta \mathrm{S} \equiv\left(\mathrm{b}_{\mathrm{S}}-\mathrm{a}_{\mathrm{S}}\right) / \mathrm{s}$.

Agora, se $n_{A}-n_{B}$ supera $\Delta S$ todos os agentes escolhem A. Os retornos esperados de $\mathrm{A}$ levam a superar a preferência natural dos agentes $\mathrm{S}$ por $\mathrm{B}$. $\mathrm{E}$ se $n_{A}-n_{B}$ for inferior a $\Delta R$, todos os agentes escolhem B. Mas no intervalo dado por

$$
\Delta \mathrm{S}<\mathrm{n}_{\mathrm{A}}-\mathrm{n}_{\mathrm{B}}<\Delta \mathrm{R},
$$

cada agente escolhe conforme sua preferência natural (os agentes de tipo $\mathrm{R}$ escolhem $\mathrm{A}$ e os agentes de tipo $\mathrm{S}$ escolhem $\mathrm{B}$ ), e as duas tecnologias podem coexistir simultaneamente. O Gráfico 1 apresenta o processo de seleção no espaço $\left(n_{A}-n_{B}, n\right)$ e divide esse espaço em três partes (em uma só A é escolhida, em outra só B é escolhida e em outra A e B são escolhidas). À guisa de exemplo assumimos que a tecnologia $\mathrm{B}$ é selecionada. 


\section{Gráfico 1 - TRAJETÓRIA DO PROCESSO DE SELEÇÃO}

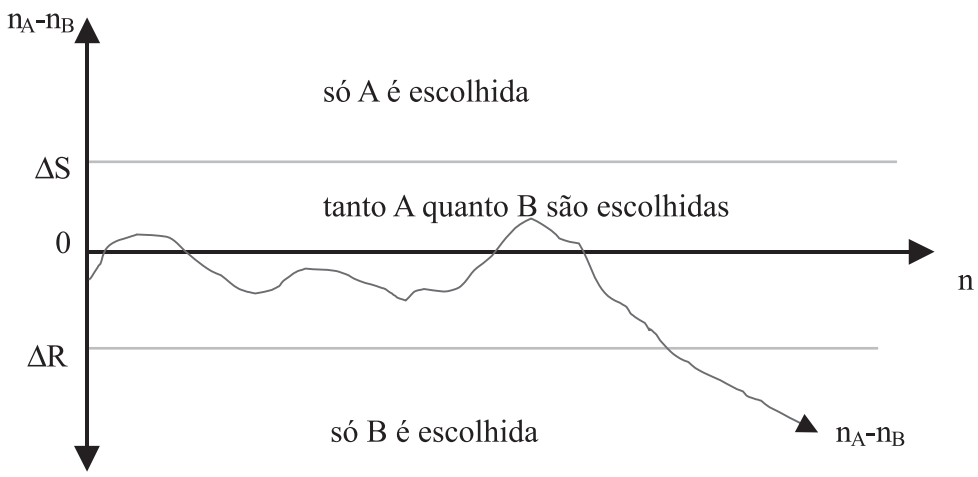

Vemos que, enquanto $n_{A}-n_{B}$ encontra-se entre $\Delta S$ e $\Delta R$, as duas tecnologias são escolhidas, mas se o processo ultrapassar DS, todos os agentes começam a escolher A, e se ultrapassar $\Delta \mathrm{R}$ todos escolhem B. Assim, os valores $\Delta \mathrm{S}$ e $\Delta \mathrm{R}$ são como barreiras que, quando superadas, dão lugar a um processo de domínio de uma das tecnologias, e, naturalmente, de exclusão da outra. Essas barreiras se denominam barreiras de absorção.

Ao ultrapassar uma barreira o processo torna-se bloqueado, o caminho é irreversível. Por exemplo, se $\mathrm{n}_{\mathrm{A}}-\mathrm{n}_{\mathrm{B}}>\Delta \mathrm{S}$, todos os agentes escolhem $\mathrm{A}$, com o qual se reforça a continuidade dessa escolha (pois $\mathrm{n}_{\mathrm{A}}$ aumenta). Arthur, et al. (1983) mostram que, neste tipo de processo, se os retornos crescentes são o suficientemente intensos, então $\mathrm{n}_{\mathrm{A}}-\mathrm{n}_{\mathrm{B}}$ deverá ultrapassar alguma das barreiras. Como resultado, a participação de uma certa tecnologia será zero ou será de $100 \%$. As duas tecnologias não podem coexistir indefinidamente e uma deve ser excluída. ${ }^{15}$

Neste tipo de sistema auto-organizativo, uma certa estrutura emerge depois de um certo número de flutuações iniciais. Das várias estruturas candidatas a serem escolhidas no longo prazo, isto é, das várias estruturas

15 É de ressaltar que o processo fica bloqueado mais rapidamente (taxa de convergência) quando as diferenças entre as preferências naturais dos agentes em relação a A e B $\left(b_{R}-a_{R}\right.$ e $\left.b_{S}-a_{S}\right)$ são menores e quando os retornos crescentes (r e s) são maiores. 
LICHA, A. L. Dependência da trajetória, irreversibilidade e o papel da história...

potenciais, a história seleciona uma, que se torna efetiva e na qual o processo fica bloqueado. ${ }^{16} 17$

Em resumo, as características principais do modelo apresentado (que ele comparte com os modelos evolucionistas em geral), são as seguintes: ${ }^{18}$

- aprendizado microeconômico imperfeito, no qual as expectativas se formam a partir do passado;

- microheterogeneidade comportamental entre os agentes, isto é, nem todos os agentes têm as mesmas preferências com relação à seleção da tecnologia, e portanto nem todos respondem igual ao mesmo conjunto de informações;

- várias formas de retornos crescentes (especialmente na acumulação de conhecimento) e não-linearidades;

- dinâmica agregada dirigida por mecanismos de aprendizado individual e de seleção coletiva;

- geração de ordem estrutural a partir de flutuações do sistema em condições de desequilíbrio.

Cada agente mantém sua decisão em função de sua preferência natural, até o momento em que os retornos crescentes provocados pela adoção de outros agentes mudam o retorno esperado pela adoção de uma tecnologia. A difusão das tecnologias, ao longo do processo seletivo, segue a tradicional curva na forma de $\mathrm{S}$, oscilando a participação das tecnologias no começo para, uma vez ultrapassada uma barreira de absorção, aumentar (diminuir) rapidamente sua participação na estrutura (convergindo, como mencionado, para $100 \%$ ou zero \% do mercado). O mercado tende a se

16 Nestes modelos não só a história (eventos acontecidos no começo) seleciona a estrutura de longo prazo. As expectativas dos agentes também podem cumprir esse papel. A este respeito ver Krugman (1991) e Arthur (1994).

17 Arthur (1994, p. 24) relaxa alguns supostos para analisar a robustez do modelo. Se o número de agentes é finito, o modelo mantém suas propriedades de absorção sempre que o número de agentes seja o suficiente grande com respeito à amplitude da brecha das barreiras absorventes. Por outro lado, se o número de agentes de cada tipo é diferente, o processo apresenta um drift. Isto desloca as barreiras de absorção, mas o processo mantém as mesmas propriedades qualitativas de longo prazo.

18 Estas características são próprias de todo processo evolucionário. Neste sentido, ver Dosi e Kaniovsky (1994). 
concentrar na tecnologia selecionada, de forma irreversível, a partir de um momento dado. ${ }^{19}$

\section{IMPLICAÇÕES PARA A POLÍTICA TECNOLÓGICA}

Da nossa análise surgem algumas implicações de política com relação a tecnologias que exibem, de forma muito significativa, retornos crescentes.

a) Como não é garantido que o processo seja eficiente do ponto de vista dinâmico, é importante manter as opções tecnológicas abertas, até que exista informação suficiente sobre as tendências futuras. Deixar que o mercado se concentre antes de ter uma idéia sobre a variedade dos resultados possíveis no longo prazo, pode levar a que o mercado fique bloqueado numa tecnologia menos eficiente. O problema, naturalmente, é como evitar a rápida concentração num processo inerentemente instável, isto é, num processo em que ela surge endogenamente, como resultado da ação das próprias forças do mercado. Como a ineficiência resulta de que nada induz aos agentes a explorar tecnologias custosas no começo, mas atrativas para futuros adotantes, o remédio natural seria oferecer aos primeiros adotantes direitos de compensação sobre o uso futuro da tecnologia. Assim, deve-se garantir aos primeiros agentes direitos de propriedade sobre parte dos lucros que a tecnologia venha a gerar no futuro; ${ }^{20}$

b) Por outro lado, Cowan (1991) mostra que se a autoridade central subsidia tecnologias com retornos crescentes, sobre a base das estimativas sobre seu rendimento futuro, é menos provável de que aconteça um bloqueio na tecnologia inferior, comparado com o caso em que não existe esse subsídio. Nos anos ' 80 este aspecto foi destacado pelo governo americano em programas como o US Strategic Defense Initiative, onde projetos com tecnologias de ponta competiam por subsídios governamentais sobre a base de resultados

19 Exceto por um número pequeno de agentes que durante o período de flutuação do processo escolheram a tecnologia que foi excluída. Se fosse adicionado um mecanismo de reavaliação das decisões dos agentes, aqueles que ficaram do lado errado poderiam mudar sua posição. Araújo (1994) apresenta um modelo com retornos crescentes onde este mecanismo é incluído.

20 Mas esses direitos devem ser aplicados sobre uma série limitada de adoções futuras para não restringir a adoção dos outros agentes. 
esperados no longo prazo. Apesar de nem sempre acontecer o bloqueio na tecnologia com potencial superior, o programa permitiu consolidar um conjunto de tecnologias que propiciaram o surgimento da chamada Nova Economia nos anos '90;

c) O timing da política é central. Como coloca Arthur (1994), existem "janelas estreitas” em que a política é efetiva, pois o subsídio (custo) necessário para induzir os agentes a mudar suas decisões numa certa direção, aumenta à medida que o processo avança. A intervenção torna-se cada vez mais cara se o processo se concentrou na direção errada;

d) Outro tema importante é o desenvolvimento e a melhora dos sistemas de informação pública em relação às tecnologias existentes, procurando antecipar seus desempenhos futuros. Órgãos de pesquisas, universidades e laboratórios públicos e privados, podem oferecer ao mercado uma perspectiva crítica dos cenários futuros de cada escolha e, assim, ajudar a evitar que a estrutura fique bloqueada numa alternativa de desempenho mais fraco no longo prazo.

\section{REFLEXÕES FINAIS}

O capítulo mostra que na escolha de tecnologias que apresentam fortes retornos crescentes a "história importa" no processo de seleção e que sua dinâmica pode apresentar várias trajetórias possíveis. Algumas qualificações adicionais ajudam a interpretar melhor a natureza da análise.

- Como supomos que o repertório de tecnologias disponíveis para os agentes é dado, podemos considerar o esquema apresentado como um modelo de difusão de tecnologias;

- Em termos de sua aplicabilidade, o modelo supõe que existe um número grande de decisões e que o processo flutua durante um certo tempo, para depois se concentrar e ficar bloqueado numa dada tecnologia;

- A análise do "momento" em que a trajetória torna-se irreversível é uma questão empírica a ser estudada em cada caso concreto;

- No modelo existe um mecanismo que promove a concentração da estrutura (gerados os retornos crescentes) e um outro mecanismo que provoca a desconcentração (devido à heterogeneidade nas preferências dos agentes). Para que o processo seja dependente da trajetória a realimentação deve ser o suficientemente intensa para provocar a exclusão de uma tecnologia. Se a heterogeneidade das preferências é tão grande que impede que isso aconteça, então o processo não apresenta as propriedades discutidas 
no texto. ${ }^{21}$ Em outras palavras, os parâmetros do modelo devem assumir certos valores para que o processo apresente as propriedades estudadas;

- Alguns autores (como Liebowitz e MARGOLIs, 1994) têm criticado estes modelos por considerar que, em geral, os retornos crescentes não são o suficientemente intensos como para gerar processos dependentes da trajetória. Segundo eles é comum que os processos sejam ergódicos, que coexistam várias tecnologias a longo prazo e que a alocação dependa das preferências dos agentes. De todas as formas, essa crítica não invalida a abordagem teórica apresentada, mas só explicita as condições empíricas nas quais seriam encontrados os fenômenos de dependência da trajetória e irreversibilidade;

- A análise sobre concorrência de tecnologias deve ser sistematicamente estudada a partir de processos dinâmicos que possuam um caráter essencialmente histórico, que nos ajudem a compreender como a seleção é efetivamente levada a cabo. Uma abordagem dinâmica nos permite entender os processos da forma como eles acontecem enquanto que uma abordagem estática não consegue incorporar o papel da história na análise econômica. ${ }^{22}$

\section{APÊNDICE: PROPRIEDAdES DO MODELO}

O modelo simplificado de seleção de tecnologias apresentado no item 4 deste capítulo possui propriedades interessantes. Analisemos elas a partir da formalização elaborada por Arthur (1989).

Como visto, o modelo tem infinitos agentes de dois tipos, $\mathrm{R}$ e $\mathrm{S}$, e duas tecnologias, A e B. As decisões dos agentes são seqüenciais: em cada "momento" um agente escolhe uma tecnologia. ${ }^{23}$ A probabilidade de escolher A ou B pode ser estabelecida, mas os agentes não sabem qual será a decisão que efetivamente acontecerá (só ex-post é possível ver as realizações do processo como uma seqüência binária de A e B). Assim, cada agente escolhe sua opção preferida, mas a seqüência em que os agentes realizam sua escolha é deixada em aberto (considerada aleatória). ${ }^{24}$

21 A respeito da tensão existente entre forças centralizadoras e descentralizadoras neste tipo de modelos pode-se ver Bassanini e Dosi (1999).

22 Neste sentido ver David (1993).

23 No modelo, tempo é o evento tempo e não o tempo histórico. Num período de tempo histórico podem acontecer vários eventos (escolhas).

24 A aleatoriedade é a expressão de alguma determinação que afeta o comportamento dos agentes mas que não é percebida pelos agentes. 
Representemos o processo pela variável de estado $\mathrm{x}_{\mathrm{A}} \equiv \mathrm{n}_{\mathrm{A}} / \mathrm{n}$ (participação da tecnologia A no total de escolhas) e analisemos como evoluciona depois que um número grande de agentes tenham realizado suas escolhas. Descrevamos o processo pela seqüência $\left\{\mathrm{x}_{\mathrm{A}}{ }^{\mathrm{n}}\right\}$, onde $\mathrm{x}_{\mathrm{A}}{ }^{\mathrm{n}}$ é a participação de A quando $\mathrm{n}$ escolhas foram feitas, e definamos formalmente as propriedades principais do processo de seleção.

\section{Previsibilidade}

Um processo é previsível se dado o grau de incerteza criado pelas flutuações iniciais, os agentes têm informação suficiente para predeterminar adequadamente a estrutura de longo prazo, isto é, se podem, ex-ante, construir uma seqüência de previsões $\left\{\mathrm{x}_{\mathrm{A}}{ }^{\mathrm{n}}\right\}$, tal que $\left|\mathrm{x}_{\mathrm{A}}{ }^{\mathrm{n}}-\mathrm{x}_{\mathrm{A}}\right| \rightarrow 0$ com probabilidade um quando $\mathrm{n} \rightarrow \infty$. O processo estudado é claramente não previsível; o observador pode prever no início do processo que no longo prazo $\mathrm{x}_{\mathrm{A}}$ será $0 \mathrm{ou}$ 1 mas não sabe qual será a tecnologia selecionada (se escolher uma tecnologia ou outra estará errando com probabilidade 0,5$){ }^{25}$

\section{Flexibilidade estrutural}

O processo é flexível se o subsídio sobre os retornos de uma tecnologia, necessário para mudar as escolhas futuras, é sempre pequeno (menor que uma constante g). No modelo, o subsídio necessário para deslocar as barreiras o suficiente aumenta sem limite. O valor crescente do subsídio indica o grau pelo qual o sistema torna-se estruturalmente rígido (lock-in). Em outras palavras, depois que o processo ultrapassou uma barreira de absorção é cada vez mais difícil mudar a estrutura gerada, já que o processo é absorvido por A ou B.

25 Neste sentido, o futuro é incerto, pois os agentes não podem conhecer o resultado de longo prazo do processo. O futuro é desconhecido e não existe forma de completar a informação a respeito do que acontecerá (pois ela não existe). Segundo Keynes (1937, p. 114), "sobre estes problemas não existe qualquer base científica para o cálculo probabilístico. Simplesmente, nada sabemos a respeito”. 


\section{Ergodicidade}

Um processo é ergódico se diferentes seqüências de eventos históricos levam com certeza à mesma estrutura de longo prazo. Mais precisamente, o processo é ergódico se dadas duas amostras do conjunto de possíveis eventos históricos $\left\{\mathrm{t}_{\mathrm{i}}\right\}$ e $\left\{\mathrm{t}_{\mathrm{i}}{ }_{\mathrm{i}}\right\}$, com as correspondentes trajetórias temporais $\left\{\mathrm{x}_{\mathrm{A}}{ }^{\mathrm{n}}\right\}$ e $\left\{\mathrm{x}_{\mathrm{A}}{ }^{{ }^{\prime}}\right\}$, então $\left|\mathrm{x}_{\mathrm{A}}{ }^{{ }^{\prime}}-\mathrm{x}_{\mathrm{A}}{ }^{{ }^{n}}\right| \rightarrow 0$ com probabilidade um quando $\mathrm{n} \rightarrow \infty$. Isto significa que eventos históricos não afetam a estrutura de longo prazo do sistema, pois o processo "esquece" esses eventos. Eles não afetam a estrutura de longo prazo, pois o processo não possui memória ou, em outras palavras, os choques aleatórios possuem apenas efeitos temporários.

Com retornos crescentes, a ordem "temporal" em que as escolhas são realizadas afeta a estrutura de longo prazo, pois escolhas realizadas no começo do processo podem levar a selecionar A ou B. A seqüência histórica de escolhas decide a trajetória da participação das tecnologias no regime de longo prazo; o processo lembra a história de pequenos eventos. O processo estudado é dependente da trajetória ou, mais precisamente, não ergódico. ${ }^{26}$

\section{Eficiência dinâmica}

No modelo proposto os atratores $\mathrm{x}_{\mathrm{A}}=1 \mathrm{e} \mathrm{x}_{\mathrm{A}}=0$ são Pareto incomparáveis, já que os agentes de tipo $\mathrm{R}$ preferem estritamente $\mathrm{x}_{\mathrm{A}}=1 \mathrm{e}$ os de tipo $S$ preferem estritamente $\mathrm{x}_{\mathrm{A}}=0 .{ }^{27}$ Por isso devemos tratar outro conceito de eficiência. Arthur (1989) define um conceito de eficiência que permite analisar se o processo seleciona a estrutura "potencialmente melhor" a longo prazo. Diremos que o processo é dinamicamente eficiente (path-efficient) se a todo momento um desenvolvimento igual (adoção igual) da tecnologia que está atrás na adoção não provocaria um retorno melhor.

A idéia é que os retornos das tecnologias podem aumentar a taxas diferentes em distintos períodos. Uma tecnologia pode apresentar baixos

26 Neste caso "a história importa" na análise do processo concorrencial.

27 No modelo, ${ }_{\mathrm{R}} \mathrm{V}\left(\mathrm{x}_{\mathrm{A}}=1\right)>{ }_{\mathrm{R}} \mathrm{V}\left(\mathrm{x}_{\mathrm{A}}=0\right) \mathrm{e}_{\mathrm{S}} \mathrm{V}\left(\mathrm{x}_{\mathrm{A}}=1\right)<{ }_{\mathrm{S}} \mathrm{V}\left(\mathrm{x}_{\mathrm{A}}=0\right)$. Logo, $\mathrm{x}_{\mathrm{A}}=1 \mathrm{e}$ $\mathrm{x}_{\mathrm{A}}=0$ não são comparáveis no sentido Pareto. $\mathrm{O}$ conceito de eficiência de Pareto não é cumprido, pois se o sistema fica bloqueado em $A$, os agentes $S$ deixam de ganhar $b_{s}-a_{s} e m$ relação a se B fosse a ação selecionada, e vice-versa. Neste sentido, ver Araújo (1994). 
retornos nas primeiras adoções, mas após ter sido adotada um certo número de vezes, pode tornar-se superior a outras tecnologias. Assim, o processo é ineficiente se fica bloqueado na tecnologia que só apresenta retornos elevados nas primeiras adoções.

Formalmente, suponhamos que U seja um indicador dos retornos efetivos de uma tecnologia e que num certo momento:

- a tecnologia A foi adotada $m$ vezes sendo seu retorno $U_{A}(m)$;

- a tecnologia B foi adotada $\mathrm{k}$ vezes, sendo $\mathrm{k}<\mathrm{m}$.

O processo é dinamicamente eficiente se:

$\mathrm{U}_{\mathrm{A}}(\mathrm{m})>\operatorname{Max}_{\mathrm{j}} \mathrm{U}_{\mathrm{B}}(\mathrm{j}), \mathrm{k}<\mathrm{j}<\mathrm{m}$

isto é, se as escolhas de $\mathrm{B}$ não teriam rendido mais que as de A. Caso contrário, teria sido melhor escolher a tecnologia B. ${ }^{28}$

Com retornos crescentes, a eficiência dinâmica da tecnologia selecionada não é garantida. Se no começo do processo uma tecnologia melhora lentamente, ela pode ser excluída e a estrutura de longo prazo pode ficar na opção inferior, pois uma adoção igual da tecnologia excluída no longo prazo teria melhores resultados para todos os agentes. A ineficiência potencial resulta do fato de que os primeiros adotantes impõem externalidades aos que chegam depois, não existindo nenhum mecanismo que induza aos agentes a explorar tecnologias custosas no começo mas atrativas para adotantes futuros. ${ }^{29} \mathrm{O}$ processo pode ficar bloqueado na tecnologia que apresenta um lucro maior nas primeiras adoções, e ser ineficiente se os rendimentos a longo prazo da outra tecnologia são maiores.

\section{Resumo}

Os processos que apresentam fortes complementaridades nas decisões dos agentes possuem as seguintes propriedades de longo prazo:

- concentração: uma tecnologia é selecionada e a outra excluída;

- não-previsibilidade (incerteza): um observador não pode prever ex ante qual será a tecnologia selecionada no longo prazo;

28 Esta definição de eficiência pode criar dificuldades empíricas e metodológicas, pois pode ser difícil de avaliar o desempenho de cada tecnologia para distintos níveis de adoção.

29 De fato, um agente pode considerar que uma tecnologia é melhor a longo prazo e escolher a outra se prevê que esta última será selecionada. 
- rigidez estrutural: a estrutura de longo prazo fica bloqueada numa tecnologia;

- dependência da trajetória: pequenos eventos históricos podem determinar a estrutura futura;

- eficiência dinâmica não garantida: o processo pode selecionar a tecnologia com retorno menor no longo prazo.

\title{
RESUMO
}

O artigo apresenta uma breve revisão dos conceitos de retornos crescentes e path-dependency e de sua importância para a teoria econômica, tomando como ponto de partida as contribuições de Brian Arthur. São discutidas algumas aplicações desses conceitos na análise de problemas específicos, especialmente na área da inovação tecnológica. É também apresentada uma versão simplificada do clássico modelo de Arthur, onde se geram situações de irreversibilidade da trajetória quando existem retornos crescentes.

Palavras-chave: economia evolucionista, retornos crescentes, path dependency.

\begin{abstract}
The paper offers a brief review of the relevance of the concepts of increasing returns and path-dependency in economic theory, taking as a starting point the contributions of Brian Arthur. The paper discusses some applications of these concepts in the analysis of specific problems, particularly in the field of technological innovation. In addition, a simplified version of the model of Brian Arthur is presented, in which lock in emerges in the presence of increasing returns.

Key-words: evolutionary economics, increasing returns, pathdependency.
\end{abstract}


LICHA, A. L. Dependência da trajetória, irreversibilidade e o papel da história...

\section{REFERÊNCIAS}

ARAÚJO, J. L. Are technology diffusion processes inherently historical? IEI/UFRJ, n. 313, 1994. Discussão.

ARTHUR, W. B. Competing technologies, increasing returns and lock-in by historical events. The Economic Journal, Mar. 1989. 99, p. 116-131.

Increasing returns and path dependence in the economy. The University of Michigan Press. 1994.

.; ERMOLIEV, Y. M. ; KANIOVSKI, Y. M. On generalized urn schemes of the Polya kind. Cybernetics, v. 16, p. 61-71. 1983.

BASSANINI, A.; DOSI, G. Competing technologies, technological monopolies, and the rate of convergence to a stable market structure. OECD, Nov. 1999. Mimeografado.

COWAN, R. Nuclear power reactors: a study in technological lock-in, Journal of Economic History, Sept. 1990. v. 50, n. 3, p. 541-567.

. Tortoises and hares: Choice among technologies of unknown merit, The Economic Journal, July. 1991. 101, p. 801-814.

.; GUNBY, P. Sprayed to death: path dependence, lock-in and pest control strategies. Economic Journal, May. 1996. v. 106, n. 436, p. 521-542.

DAVID, P. A. Clio and the economics of QWERTY. American Economic Review, May. 1985. v. 75, n. 2.

. Path dependence and predictability in dynamic systems with local network externalities: a paradigm for historical economics. In: D. FORAY ; C. FREEMAN. (Eds.). Technology and the Wealth of Nations. London: Pinter Publishers, 1993.

. Path dependence, its critics and the quest for historical economics. Stanford CA: Stanford University Economics Department, June. 2000.

.; BUNN, J. The economics of gateway technologies and network evolution: lessons from electricity supply history. Information Economics and Policy, v. 3, p. 165-202, 1988.

DOSI, G. e KANIOVSKI, Y. M. On 'badly behaved' dynamics: Some applications of generalizes urn schemes to technological and economic change. Journal of Evolutionary Economics, vol. 4, p. 93-123, 1994.

DURLAUF, S.N. Nonergodic economic growth. Review of Economic Studies, 60, p. 349-366, 1993.

FORAY, D. e GRÜBLER, A. Morphological analysis, diffusion and lock-out of technologies: ferrous casting in France and the FRG. Research Policy, v. 9, p. 535$550,1990$. 
KEYNES, J. M. (1937), The general theory of employment. In: MOGGRIDGE, D. (Ed.). The Collected Writings of John Maynard Keynes, v. 14, Macmillan London, 1973.

KRUGMAN, P. History versus expectations. Quarterly Journal of Economics: p. 651-667, 1991.

LIEBOWITZ, S.; MARGOLIS, S. Network externality: an uncommon tragedy. Journal of Economic Perspectives, 8 (2), p. 133-150, Spring. 1994.

MILGROM, P.; QIAN, Y.; ROBERTS, J. Complementarities, momentum, and the evolution of modern manufacturing. The American Economic Review, v. 81, n. 2, p. 84-88, 1991.

NORTH, D. Institutions, institutional change and economic performance. Cambridge: Cambridge University Press, 1990. 